\title{
UNA ALERTA LOEWENSTENIANA SOBRE EL USO DE LAS HERRAMIENTAS CONSTITUCIONALES DE PODER: ACLARACIONES SOBRE LAS LEYES CON DECLARATORIA DE URGENTE CONSIDERACIÓN
}

\author{
Luis Fleitas de León*
}

\begin{abstract}
RESUMEN. A partir de una nota periodística realizada a uno de los precandidatos a Presidente de la República, en las elecciones que se celebrarán en el año 2019, se analiza la herramienta constitucional de las Leyes que cuentan con una declaratoria de urgente consideración por parte del Poder Ejecutivo, con el fin de exponer, desde una perspectiva 'loewensteniana', los efectivos límites que la Constitución establece al Poder Ejecutivo para el ejercicio de esta potestad declaratoria, particularmente, en cuanto al posible contenido de estas Leyes y a su necesaria relación con una situación de existencia fáctica crítica o urgente.
\end{abstract}

PALABRAS CLAVE. Ley. Urgente. Poder Ejecutivo. Limites.

ABSTRACT. On the basis of a journalistic report made to one of the precandidates to the Presidency of the Republic, in the elections to be held in 2019, we analyze the constitutional tool of Laws that have a declaration of urgent consideration by the Executive Power, for the purposes to expose, from a 'Loewenstenian' perspective, the effective limits that the Constitution establishes to the Executive Power for the exercise of this declaratory power, particularly, as for the possible content of these Laws and their necessary relationship with an urgent or critical situation.

KEY WORDS. Law. Urgent. Executive Power. Limits.

\section{PLANTEO}

En nota publicada el día Domingo 22 de octubre de 2018, en el diario El País, uno de los precandidatos a la Presidencia de la República, no importa quién, ni importa el lema o

* Doctor en Derecho y Ciencias Sociales, Universidad de la República. Profesor Agregado de Derecho Constitucional (Grado 3 contratado) Facultad de Derecho, Universidad de la República. Correo electrónico: luisflei@hotmail.com. 
partido político, declaró: "Vamos a necesitar un Poder Ejecutivo con un poder de decisión muy fuerte. No tiene que ser uno de consulta, tiene que ser de peso político”, agregando posteriormente: "El gobierno empieza en la transición. Y allí vamos a tener una gran ley de urgencia donde ingrese todo lo que haya que modificar del Estado, salvo de lo que sea materia exclusiva de la ley presupuestal".

Esta declaración sirve como impulso para analizar la herramienta constitucional de las Leyes con declaratoria de urgente consideración, no con el objetivo de evaluar la conveniencia política de su eventual utilización, sino, con el fin de desentrañar, desde una perspectiva 'loewensteniana', los efectivos límites que la Constitución establece al Poder Ejecutivo para el ejercicio de esta potestad, particularmente, en cuanto a su contenido.

Establecer con precisión estos límites y sus razones, son la clave para el ejercicio responsable, no excedido y ajustado a la Constitución de esta particular herramienta de poder por parte del gobierno de turno.

\section{ORIGEN Y CONTEXTO.}

El mecanismo de la Ley con declaratoria de urgente consideración fue incluido, por vez primera, en nuestra Constitución, con la reforma de 1966, como un procedimiento extraordinario o diferente al normal de sanción de las Leyes, con una fuerte intervención e incidencia del Poder Ejecutivo.

Su inclusión tiene la particularidad que, a pesar de ser un procedimiento especial para la sanción de una Ley, su inserción no fue en la Sección V de la Constitución relativa al Poder Legislativo, sino en el Artículo 168, el cual establece las facultades esenciales del Poder Ejecutivo.

Lo señalado pone de manifiesto que este instituto de las Leyes con declaratoria de urgente consideración se inscribe en la innegable orientación que tuvo la Constitución de 1967 de fortalecer el Poder Ejecutivo en pos de su mayor eficacia activa ${ }^{2}$; que se inscribe en una visión propia de la época, claramente trasuntada por SANGUINETI y PACHECO SERÉ en su breve ensayo sobre esta Constitución, al justificar con una lapidaria frase, las razones que se entendían para tal fortalecimiento: "de la constitución del Poder Ejecutivo, especialmente depende la suerte de los Estados de América del Sur”’.

Complementariamente, existía también la concepción de que el Poder Legislativo demostraba cierta ineficacia para resolver determinados items o necesidades o exigencias cuya perentoriedad requieren una resolución legal rápida, idea que tuvo clara incidencia en la incorporación de las Leyes con declaratoria de urgente consideración por parte del Poder Ejecutivo ${ }^{4}$, por las razones que veremos.

1 Disponible: https://www.elpais.com.uy/informacion/politica/luis-lacalle-pou-compartir-sindicatos.html Consulta: 19/11/2018.

2 CASSINELLI MUÑOZ, Horacio; "Primeras reflexiones sobre la nueva Constitución” en Derecho Constitucional y Administrativo, $1^{a}$ edición, Montevideo, La Ley Uruguay, 2010, pp. 123, $124,131$.

3 SANGUINETTI, Julio, PACHECO SERÉ, Álvaro. La nueva Constitución, Alfa, Montevideo, pp. 165.

4 REAL, Alberto Ramón. "Las Leyes con declaratoria de urgente consideración”. En CORREA FREITAS, Ruben, El cincuentenario de la Constitución uruguaya de 1967, Montevideo, Universidad de la Empresa, Grupo Magro Editores, 2017, p. 85. 
Si bien esta fue la orientación de la Constitución de 1967, esta herramienta no implica, por si misma, un desequilibrio entre los Poderes, en particular respecto del Poder Legislativo $^{5}$. Sin embargo, esta clase de facultades requiere un ejercicio responsable por parte de quienes expresan la voluntad el Poder Ejecutivo, puesto que, como señaló uno de los padres del constitucionalismo moderno, el alemán Karl LOEWENSTEIN, también en la década del sesenta del siglo pasado: "el poder lleva en sí mismo un estigma y sólo los santos entre los detentadores del poder - ¿y donde se pueden encontrar?- serían capaces de resistir a la tentación de abusar del poder"‘ .

Esta clase de poderes, la declaratoria de urgente consideración, que como veremos generan una situación exhorbitante en el procedimiento de sanción de una Ley que tiende a favorecer la voluntad del gobierno que la impulsa, están previstos para su ejercicio en ciertas situaciones que así lo justifiquen y respetendo la esencia de los Poderes, que es lo que explica que no exista un desequilibrio al menos formalmente. El ejercicio responsable de un poder implica hacerlo dentro de los límites y con el própósito que la Constitución establece, evitándo así el abuso de parte de quien ejerce el poder o quizás, de modo más sutil, la tentación del abuso.

\section{POSIBLES CONTENIDOS DE ESTAS LEYES: LÍMITES AL PODER DE CLARATORIO DEL EJECUTIVO.}

El Artículo 168 numeral $7^{\circ}$ de nuestra Constitución confiere al Poder Ejecutivo la facultad de emitir la declaratoria de urgente consideración de una Ley, en realidad de un proyecto de Ley, la que deberá ser hecha al remitir el proyecto al Poder Legislativo. Esta declaratoria sólo puede ser emitida por el Presidente de la República actuando en Consejo de Ministros, conforme al Artículo 160 de la Constitución y no en acuerdo con algunos Ministros que es la otra modalidad de actuación de Poder Ejecutivo.

La Ley con declaratoria de urgente consideración puede tener contenido múltiple y variado, pero también único. Sin perjuicio de ello, la Constitución establece tres limites acumulativos a la potestad del Ejecutivo de efectuar la referida declaratoria ${ }^{7}$, que ofician de control ante un eventual ejecicio desbordado de esta facultad exhorbitante.

En primer lugar, no pueden recibir la declaración de urgente consideración las Leyes de Presupuesto (literal b del Artículo).

En segundo lugar, no pueden recibir declaración de urgente consideración las Leyes que requieran para su aprobación 2/3 o 3/5 de votos del total de componenetes de cada Cámara (literal b del Artículo), que son las Leyes que según el constituyente tienen más importancia política $^{8}$, y sí las que requieren mayoría simple y absoluta.

5 LOEWEnstein, Karl. Teoría de la Constitución, reedición de abril de 2018 de la edición de 1965, Barcelona, Editorial Ariel, p. 269

6 LOEWENSTEIN, Karl; op. cit., p. 28.

7 Adicionalmente, el literal a) del Artículo 168 numeral $7^{\circ}$ de la Constitución prevé otro límite al Poder Ejecutivo, pero que no es al contenido u objeto de esta clase de Leyes, sino que consiste en que éste "no podrá enviar a la Asamblea General más de un proyecto de ley con declaratoria de urgente consideración simultáneamente, ni enviar un nuevo proyecto en tales condiciones mientras estén corriendo los plazos para la consideración legislativa de otro anteriormente enviado".

8 CASSINELLI MUÑOZ, Horacio. Derecho Público, edición de julio 2009, $3^{\mathrm{a}}$ actualización, Montevideo, Fundación de Cultura Universitaria (FCU), p. 228. 
En tercer lugar, el límite más sutil y en el que debe tenerse mayor precaución por parte del gobernante ejecutivo, surge de la propia nomenclatura que utiliza el numeral $7^{\circ}$ del Artículo 168 de la Constitución, al decir que se trata de Leyes "con declaratoria de urgente consideracion", y no simplemente de "urgente consideración" como se las ha llamado de forma algo descuidada, incluso, por cierta literatura jurídica.

El uso del término "declaratoria”, refiere a una declaración, a algo declarativo, que se opone conceptualmente a algo que es constitutivo. Esa es la clave. Con precisión, KORZENIAK señala que, en cuanto al contenido de estas Leyes, "los temas regulados deben tener cierta razonable urgencia de ser resueltos. Esta no es sólo una consideración de sentido común, sino que se deriva del carácter declarativo de la urgencia”. El Poder Ejecutivo "no debe inventar o constituir la urgencia del asunto", por razones políticas o de concepción ideológica o por prioridades de gobierno, muy por el contrario, "formula una declaración de una urgencia realmente existente ${ }^{\text {, }}$, debe declarar una urgencia con alguna base fáctica razonable, sea económica, sanitaria, social e incluso estatal.

A riesgo de ser reiterativo, intentaré ser más preciso. El término “declaratoria” tiene dos sentidos que se intersectan, por un lado, significa la manifestación de la voluntad del Poder Ejecutivo, la acción de declarar, pero además, significa que la manifestación de voluntad debe ser declarativa -y no constitutiva- de una situación de urgencia realmente existente, que es el presupuesto de hecho y el motivo validante de la manifestación del Poder Ejecutivo en estos casos.

Particularmente es este límite el que impide que el gobernante ejecutivo que tiene mayoría parlamentaria, sea propia o por coalición, y que es usual que la tenga dados los caracteres de nuestro sistema electoral y político ${ }^{10}$, gobierne al impulso volutarista de esta clase de Leyes de aprobación sumaria, pues ellas tienen el sólo propósito de constituirse en una acción eficaz y puntual ante situaciones críticas y urgentes ${ }^{11}$.

Atendiendo al criterio finalista de interpretación de la Constitución ${ }^{12}$, es este el modo en el que debe inteligirse la disposición constitucional en cuestión, pues, como señalaba LOEWENSTEIN, ontológicamente “... se debe considerar como 'telos' de toda Constitución -y no hay motivo para que la nuestra sea una excepción-, la creación de instituciones para limitar y controlar el poder político, ... limitar el poder político quiere decir limitar a los detentadores del poder", como único modo de exorcisar la tentanción del abuso ${ }^{13}$.

9 KORZENIAK, José. Primer Curso de Derecho Público. Derecho Constitucional, $3^{\mathrm{a}}$ edición, Montevideo, FCU, febrero de 2006, p. 491.

10 Véase que desde que se instauró el sistema electoral de ballotage', en la reforma constitucional de 1996 , los Ejecutivos, en mayor o menor medida, sean porpias como en el caso de los gobiernos del Frente Amplio o coaligadas como en el caso de los gobiernos del Partido Colorado, siempre han contado con mayorías en el Parlamento.

11 Nobleza obliga, cabe señalar que, de forma no expresa y de modo extrañamente acrítico, CASSINELLI parecería exponer otro criterio, al señalar que las leyes con declaratoria de urgente consideración aseguran al Poder Ejecutivo el pronunciamiento en plazos razonables acerca "de una ley que considere necesaria para la ejecución de su política” - CASSINELLI, Horacio, Derecho Público, op. cit., p. 228.

12 JIMENEZ DE ARÉCHAGA, Justino. La Constitución Nacional, edición de la Cámara de Senadores de 1988, reedición de 1991, Montevideo, Tomo I, pp. $134-139$.

13 LOEWENSTEIN, Karl, op. cit., pp. 29, 151. Decía el autor: "La historia del constitucionalismo no es sino la búsqueda por el hombre político de las limitaciones al poder absoluto...” (p. 150) 
Dicho esto entonces, la expresión del precandidato: “... allívamos a tener una gran ley de urgencia donde ingrese todo lo que haya que modificar del Estado”, podría transuntar al menos una concepción excedida de esta facultad constitucional, pues esto no obedece a la declaración de una urgencia existente en el Estado, a una situación crítica, sino a una cuestión de concepción del mismo, lo que es absolutamente legítimo, pero no es de por sí hábil para motivar tal declaratoria.

Dicho de un modo crudo, ni la mejor y más inspirada reforma del Estado podría efectuarse por una Ley con declaratoria de urgente consideración, salvo que tuviese como presupuesto fáctico una situación estatal crítica generalizada, extremo que, más allá de los cuestionamientos que pueden realizarse al Estado uruguayo, parece no verificarse.

\section{PRERROGATIVAS DE ESTAS LEYES: LA IMPORTANCIA DE LOS LÍMITES AL PODER DECLARATORIO.}

El procedimiento de sanción de estas Leyes declaradas de urgente consideración por parte del Poder Ejecutivo, tienen una serie de prerrogativas exhorbitantes, si se las compara con el de las Leyes comúnes, que dimensionan explícitamete la relevancia del ejercicio limitado tal potestad por quienes expresan la voluntad del Poder Ejecutivo.

En primer lugar, el Poder Legislativo tiene plazos breves "casi conminatorios"14 para la sanción de estas Leyes: la Cámara que recibe proyecto tiene 45 días para tratarlo, si no lo desecha expresamente se reputa aprobado tal cual lo envió el Poder Ejecutivo (literal d); la segunda Cámara tiene 30 días para tratarlo y si no media pronunciamiento expreso de desecho también queda aprobado el proyecto como fue enviado por el Poder Ejecutivo (literal e); si la segunda Cámara aprueba un texto distinto, debe reenviarlo a la primera que dispone de 15 días (literal e); si la primera Cámara no se pronunciare sobre el texto modificado por la segunda Cámara, el proyecto se debe remitir de oficio ante la Asamblea General que dispone de 10 días para su consideración, la que podrá dirimir la diferencia entre ambas Cámaras y pronunciarse expresamente incluso aprobando un nuevo proyecto por 2/3 de votos o no pronunciarse, en cuyo caso se tendrá por sancionado el proyecto en la forma en la cual lo votó la última Cámara que le prestó aprobación expresa (literales e, f y Artículo 135).

Véase que los proyectos de Ley comunes no tienen plazo para su tratamiento, salvo los proyectos de Ley que, no siendo de Presupuesto o de Rendición de Cuentas, les es aplicable el Artículo 229 de la Carta, en cuyo caso el plazo no es para su tratamiento por cada Cámara o por la Asamblea General, sino para su sanción, que debe ocurrir doce meses antes de la fecha de las elecciones ordinarias.

En segundo lugar, relacionado con lo anterior, el carácter más exhorbitante de estas leyes es que el silencio de los órganos del Poder Legislativo, luego de transcurridos los plazos que se indicaron, implican la sanción de la Ley en cuestión. Es decir, la Ley se puede aprobar con la sola voluntad expresa positiva del Poder Ejecutivo.

En los proyectos de Ley comunes, la falta de pronunciamiento de los órganos del Poder legislativo tiene por efecto el rechazo o el archivo, nunca la aprobación tácita.

${ }^{14}$ KORZENIAK, José, op. cit, p. 492. 
Algunos autores intentan atenuar el carácter exhorbitante de esta clase de Leyes, lícita por supuesto, pero exhorbitante al fin, invocando ciertas similitudes con las Leyes de Presupuesto y de Rendición de Cuentas, en cuanto a que el Parlamento debe pronciarse en determinados plazos ${ }^{15}$ y a que ambas tienen inicitativa privativa del Poder Ejecutivo.

Sin embargo, existe una importante diferencia.

Por un lado, si bien ambas Cámaras tienen plazos para tratar el proyecto de Ley de Presupuesto o Rendición, los términos son un poco más extensos que los de las Leyes con declaratoria de urgente consideración: 45 días para cada Cámara, 15 días en caso que se introduzcan modificaciones y si interviene la Asamblea General, también 15 días más (Artículos 217 y 218).

Por otro lado, la diferencia sustancial con las Leyes con declaratoria de urgente consideración es que en caso que las Cámaras y eventualmente la Asamblea General, no se pronunciaren en los plazos indicados, el proyecto de Ley de Presupuesto o Rendición se tendrá por rechazado (incisos $2^{\circ}$ de los Artículos 217 y 218).

Finalmente, la única herramienta de contrapeso para eclipsar al Poder Ejecutivo, que para estos casos la Constitución prevé en favor del Poder Legislativo, es que con el voto conforme de 3/5 del total de componentes de cada Cámara se puede dejar sin efecto la declaratoria de urgente consideración (literal c del numeral $7^{\circ}$ Artículo 168).

Ahora bien, la mayoría calificada de 3/5 para ejercer el contrapeso, no tiene por objetivo proteger la voluntad de las minorías, que es la razón final que normalmente tiene de este tipo de mayorías ${ }^{16}$, sino proteger la propia voluntad del Poder Ejecutivo ${ }^{17}$ en su declaración, que, como ya se dijo, en nuestro sistema de gobierno tiende a ser respaldada por una mayoria parlamentaria propia o coaligada que facilmente supera el quantum de 2/5 de componentes de los órganos legislativos, lo que atenúa manifiestamente la eficacia de este contrapeso legislativo.

\section{CONCLUSIÓN.}

Las prerrogativas innegables que implica la declaratoria de urgente consideración de un proyecto de Ley y el disminuído contrapeso del Poder Legislativo a la voluntad del Poder Ejecutivo, dimensionan la prudencia, razonabilidad y el estricto ajuste a la Constitución con que deben actuar quienes expresan jurídicamente la voluntad el Poder Ejecutivo en estos casos, el Presidente de la República con el Consejo de Ministros; en particular, guardando un vínculo estricto entre la emisión de dicha voluntad y una realidad fáctica crítica 0 urgente, como el presupuesto de hecho y motivo válido de tal declaración.

El ejercicio de este poder sin contemplar lo señalado, sin que la declaratoria del Poder Ejecutivo refleje una realidad fáctica crítica, urgente o de emergencia, supone una

15 CORREA FREITAS, Ruben. Derecho Constitucional Contemporáneo, $5^{\mathrm{a}}$ edición, Montevideo, FCU, noviembre 2016, Tomo II, p. 86.

16 LOEWENSTEIN, Karl, op. cit., p. 245. El autor dice: "Mayorías calificadas son una técnica importante de los controles intraórgano, por medio de los cuales el poder estar debidamente distribuído, y se alcanzará una eficaz protección de la minoría limitando a la mayoría".

17 CASSINELLI MUÑOZ, Horacio, Derecho Público, op. cit., p. 228. 
discordancia entre el Artículo 168 numeral $7^{\circ}$ de la Constitución y el ejercicio del poder exhorbitante que contiene, nominalizando así peligrosamente el elemento de control que prevé la norma constitucional como límite al gobernante ejecutivo ${ }^{18}$.

Todo indicio de ello, por más eventual que sea, debe ser objeto de alerta.

\section{BIBLIOGRAFÍA CONSULTADA.}

CASSINELLI MUÑOZ, Horacio. Derecho Público, edición de julio 2009, $3^{\text {a }}$ actualización, Montevideo, Fundación de Cultura Universitaria.

CASSINELLI MUÑOZ, Horacio; "Primeras reflexiones sobre la nueva Constitución" en Derecho Constitucional y Administrativo, $1^{a}$ edición, Montevideo, La Ley, 2010.

CORREA FREITAS, Ruben. Derecho Constitucional Contemporáneo, $5^{\mathrm{a}}$ edición, Montevideo, Fundación de Cultura Universitaria, noviembre de 2016, Tomo II.

JIMENEZ DE ARÉCHAGA, Justino. La Constitución Nacional, edición de la Cámara de Senadores de 1988, reedición de 1991, Montevideo, Tomo I.

KORZENIAK, José. Primer Curso de Derecho Público. Derecho Constitucional, $3^{\text {a }}$ edición, Montevideo, FCU, febrero de 2006, p. 491.

LOEWENSTEIN, Karl. Teoría de la Constitución, reedición de abril de 2018 de la edición de 1965 , Barcelona, Editorial Ariel.

REAL, Alberto Ramón. "Las Leyes con declaratoria de urgente consideración", En CORREA FREITAS, Ruben, El cincuentenario de la Constitución uruguaya de 1967, Universidad de la Empresa, Grupo Magro Editores, 2017.

SANGUINETTI, Julio, PACHECO SERÉ, Álvaro; La nueva Constitución, editorial Alfa. Montevideo.

Fecha de recepción: 20 enero 2019.

Fecha de aceptación: 12 de marzo 2019.

18 LOEWENSTEIN, Karl, op. cit., pp. 216 - 218. En su clasificación ontológica de las Constituciones, el autor distingue entre Constituciones normativas y nominativas, además de las semánticas. Las normativas son aquellas jurídicamente válidas, que además, son efectivamente vividas por destinatarios y detentadores, son reales y efectivas y acompañadas por la realidad del ejercicio del proceso de poder. Las nominativas son Constituciones jurídicamente válidas, pero a cuyas normas no se adapta la dinámica del proceso del ejercicio del poder político, dejando sin realidad existencial a la misma más allá de su existencia en el plano jurídico. 
